\title{
L-Isoleucine in a Choline Chloride/Ethylene Glycol Deep Eutectic Solvent: a Reusable Reaction Kit for the Asymmetric Cross-Aldol Carboligation
}

\author{
Noé Fanjul-Mosteirín, Carmen Concellón*, Vicente del Amo* \\ Departamento de Química Orgánica e Inorgánica, Universidad de Oviedo, C/ Julián Clavería 8, 33006, \\ Oviedo, Spain. E-mail: ccf@uniovi.es; vdelamo@uniovi.es
}

\begin{abstract}
L-Isoleucine is able to catalyze the cross-aldol reaction between cyclohexanone and aromatic aldehydes in a deep eutectic solvent consisting in choline chloride and ethylene glycol, rendering products with high diatereo- and enantioselectivity. This protocol is straightforward and green: the organocatalyst and the reaction medium can be recycled up to five times, allowing the preparation of different substrates with a single load of solvent and catalyst.
\end{abstract}

The independent discoveries of List, Lerner and Barbas, ${ }^{1}$ and MacMillan, ${ }^{2}$ in 2000, put forward how small organic molecules are able to catalyze organic transformations with optimum levels of selectivity. Since then, the interest of the scientific community in the so-termed field of asymmetric organocatalysis has increased rapidly, new catalysts and organocatalyzed reactions being discovered at a breathtaking pace.

Research dealing with organocatalysis is particularly attractive for the industry, as it is considered a green technology. The concept of Green Chemistry refers to actions aimed to improve the efficiency in the use of natural resources, comprising the design and implementation of new chemical processes and transformations operating in a more efficient, safer, and more environmentally friendly way. In order to evaluate the "greenness" of a certain process, Green Chemistry has been formulated according to twelve universal principles. ${ }^{3}$ Organocatalytic transformations satisfy several of them: high atomic efficiency, low generation of residues, and use of reagents in catalytic amounts, among others. However, as a drawback, most of these practices imply the use of organic solvents.

With the intention of developing yet more sustainable processes, deep-eutectic solvents (DESs) have been recently introduced as environmentally benign reaction media, capable of pushing aside hazardous volatile organic solvents. First reported in 2001 by Abbot and co-workers, ${ }^{4}$ DESs typically consist in a combination of an electron neutral hydrogen-bond donor unit (HBD) and an ammonium-, or phosphonium-based, salt. The HBD partner is able to establish a complex network of strong hydrogen-bonding interactions with the anion (commonly a halide anion) of the salt. Such interactions organize DESs according to a layered sandwich-like structure, ${ }^{5}$ which is ultimately responsible of the unique physicochemical properties of this kind of fluids, mainly: (a) low glass transition temperature at a specific molar ratio of salt to $\mathrm{HBD},{ }^{6}$ affording DESs with melting points lower than either of their individual components; (b) low vapor pressure, therefore avoiding vapor emissions to the atmosphere; (c) an easily tunable nature; (d) compatibility with water. Furthermore, both the ammonium salt (commonly choline chloride) and the HBD units (mainly ethylene glycol, glycerol, urea, lactic acid) required to form the DESs are costless, and readily-available, biodegradable and non-cytotoxic. ${ }^{7}$

Considering the advantages listed above, DESs are starting to encounter extensive applications in metal-catalyzed organic transformations, ${ }^{8}$ as well as in enzymatic processes. ${ }^{9}$ In contrast, to the best of our knowledge, only few examples have been recently reported dealing with organocatalytic transformations operating in a DES-based reaction media. ${ }^{10}$ Following our interest in environmentally-friendly organocatalysis, ${ }^{11}$ herein we report an exhaustive study on the asymmetric crossaldol reaction catalyzed by a natural primary amino acid, Lisoleucine, carried out in a benign deep-eutectic solvent consisting of choline chloride and ethylene glycol.

The aldol reaction is one of the most renowned transformations in organic synthesis, privileged as a carboligation process for assembling the backbone of complex organic molecules. ${ }^{12}$ This $\mathrm{C}-\mathrm{C}$ bond forming reaction is receiving particular attention since the advent of organocatalysis, ${ }^{13}$ being nowadays considered as an standard playground for testing the chemical behavior of novel chiral organocatalysts and their methodologies. In this sense, in order to study the feasibility of DESs as reaction media in organocatalyzed processes, we adopted the reaction of Scheme 1 as a case of study.

Scheme 1. Case of study for the asymmetric cross-aldol reaction in $[\mathrm{ChCl} / 2 \mathrm{EG}] .^{a}$
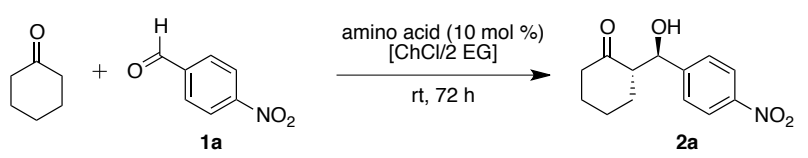

A Deep Eutectic Solvent consisting of a 1:2 molar mixture of choline chloride $(\mathrm{ChCl})$ and ethylene glycol (EG) was chosen as reaction medium, considering that this specific compo- 
sition of DES had not been previously explored in other works dealing with organocatalysis. ${ }^{10} \mathrm{ChCl}$ and $\mathrm{EG}$ are both rather inexpensive and they are meant to present very low toxicity. Looking for developing a green methodology amenable of being adopted by a broad audience readily accessible off-thebench proteinogenic amino acids were thought as potential chiral catalysts (Supporting Information (SI), Table S1). The effectiveness of the organocatalysts was evaluated in terms of conversion, diastereoselectivity and enantiomeric excess of product 2a. Interestingly, L-isoleucine provided the best results, with an ee peaking at $96 \%$, favoring the $(2 S, 3 R)$ enantiomer of adduct $\mathbf{2 a}$. It is worth noting that the isoleucinecatalyzed cross-aldol reaction between cyclic ketones and aromatic aldehydes has received little attention in classical methodologies that employ organic solvents. ${ }^{14}$ In this protocol, L-proline fails in providing competitive selectivities (SI, Table S1, entry 1), in agreement with the work of Guillena and Ramón and their study of this catalyst in ChCl-based DESs. ${ }^{10 \mathrm{c}}$

\section{[Please insert Table 1 here]}

To our delight, picking out L-isoleucine as the organocatalyst of choice for the cross-aldol reaction in $\mathrm{ChCl} / \mathrm{EG}$, an exhaustive screening of reaction parameters (catalyst loading, concentration, reaction temperature, and reaction time; see SI, Tables S2-S8) revealed an ideal setup: when a solution of 4-nitrobenzaldehyde 1a (1.0 equiv), Lisoleucine $(20 \mathrm{~mol} \%)$ cyclohexanone (10.0 equiv) and distilled water ${ }^{15}$ (10.0 equiv), in $\mathrm{ChCl} / \mathrm{EG}(1: 2, c=0.25 \mathrm{M})$, was vigorously stirred for $90 \mathrm{~h}^{16}$ at $20{ }^{\circ} \mathrm{C}$ (controlled by a thermostatic bath), the corresponding aldol adduct 2a was rendered in full conversion, with good anti-diastereoselectivity (90:10 anti/syn) and excellent enantioselectivity (98\% ee, for (2S,3R)-anti-2a) (Table 1, entry 1). The reaction is carried out in a closed-cap test tube, under air, with no special care against moisture. In spite of its substantial polarity, the aldol adduct 2 a could be fully extracted from the eutectic solvent with portions of ethyl acetate (EtOAc), being purified afterwards by flash chromatography. Interestingly, when the reaction between cyclohexanone and aldehyde 1a was run under analogous conditions, but using pure EG as the solvent, the corresponding aldol product $\mathbf{2 a}$ was produced in very low conversion, featuring modest diastereo- and enantioselectivity (Table 1, entry 2), hence manifesting the positive cooperative effect of the DES and the chiral catalyst. Another blank experiment confirmed that the DES itself, without isoleucine, does not suffice to catalyze the reaction (Table 1, entry 3 ).

With ideal conditions in hand, to establish the scope of our aldol protocol a selection of aldehydes $\mathbf{1 b}$-k bearing diverse functional groups and substitution patterns were reacted with cyclohexanone. All the reactions shown in Table 1 proceeded smoothly: aldols $\mathbf{2 b - i}$, decorated with nitro, nitrile, amide, or halide substitutents were isolated in good yield, and with high diastereo- and enantioselectivity (Table 1, entries 4-11). 4-Carboxymethyl benzaldehyde $\mathbf{1} \mathbf{j}$, was conveniently converted into the corresponding $\beta$-hydroxyketone $\mathbf{2} \mathbf{j}$ with no hydrolysis or transesterification of the ester function (Table 1, entry 12). Interestingly, aldol $\mathbf{2 k}$, derived from pyridine 2-carboxaldehyde, a challenging substrate in the aldol reaction, was isolated in fair yield (Table 1, entry 13). Moreover, isobutyraldehyde also proved to be an acceptable substrate for the cross-aldol reaction with 4-nitrobenzaldehyde 1a, giving rise to the aldol adduct $\mathbf{3 a}$ in good yield, although with modest enantioselectivity (Table 1, entry 14). In either case, such an ee value for $\mathbf{3 a}$ is comparable to the one previously reported in the literature for the same product, prepared by a cross-aldol methodology that employs $50 \mathrm{~mol} \%$ of L-isoleucine in DMSO. $^{17}$

At this stage, the recyclability of the DES containing L-isoleucine was assessed. To this end, a reaction between cyclohexanone and 4-nitrobenzaldehyde, 1a, was set up as in Table 1, entry 1 . With the completion of the reaction (full conversion), the crude mixture was treated with several portions of EtOAc $(3 \times 1 \mathrm{~mL})$, being decanted each time. The organic phases were collected together and evaporated under reduced pressure to afford product 2a quantitatively. L-Isoleucine, presumably as its zwitterionic form, remained solubilized in the $\mathrm{ChCl} / \mathrm{EG}$ DES, and was not transferred into the organic solvent. The DES medium was further subjected to vacuum to remove rests of EtOAc, cyclohexanone or water, before it was reused in subsequent reactions. By these means, the DES could be reused up to 5 times, upon addition of fresh reagents (aldehyde 1a, ketone, and water), without jeopardizing the conversion or the enantioselectivity of $\beta$-hydroxy ketone 2a, but with a slight decrease of its diastereoselectivity from the fifth catalytic cycle, probably due to some coextraction of L-isoleucine and the concomitant decrease of catalyst loading (Table 2). After the fifth run L-isoleucine displays a catalytic turn over number (TON) of over 24 units, which is a convenient figure for an organocatalyzed transformation.

Table 2. Recyclability of DES and L-isoleucine in the asymmetric cross-aldol reaction between cyclohexanone and 4-nitrobenzaldehyde 1a. ${ }^{a}$

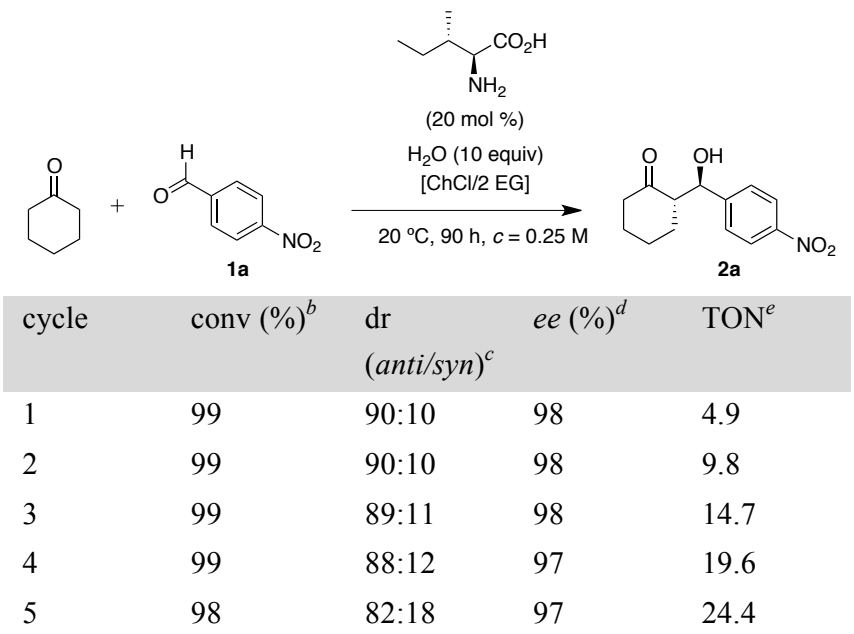

${ }^{a}$ Reaction conditions: aldehyde $1 \mathrm{a}(0.2 \mathrm{mmol})$, cyclohexanone $(2.0 \mathrm{mmol})$, distilled water $(2.0 \mathrm{mmol})$ and $\mathrm{L}$-isoleucine $(0.04$ $\mathrm{mmol})$ were added to $[\mathrm{ChCl} / 2 \mathrm{EG}](0.8 \mathrm{~mL})$, at $20{ }^{\circ} \mathrm{C}$. The reaction mixture was stirred for $90 \mathrm{~h}$. Recycling was performed as stated in the main text. ${ }^{b}$ Conversion of aldehyde 1a to anti- and syn-2a, as determined by ${ }^{1} \mathrm{H}$ NMR spectroscopy of crude reaction mixtures. Resonances of $\mathbf{2 a}$ were integrated and quantified against $\mathrm{CHBr}_{3}(9 \mu \mathrm{L}, 0.103 \mathrm{mmol})$ used as analytical internal standard. ${ }^{c}$ Diastereisomeric ratio (dr) determined by ${ }^{1} \mathrm{H}$ NMR spectroscopy from crude reaction mixtures. ${ }^{d}$ Enantiomeric excess of major diastereoisomer (anti), as determined by chiral HPLC on crude reaction mixtures. ${ }^{e}$ Accumulative turn over number (TON) values $(\mathrm{TON}=$ mol product $/ \mathrm{mol}$ catalyst $)$. 
Table 1. Scope of the cross-aldol reaction catalyzed by L-isoleucine in [ChCl/2 EG]. ${ }^{a}$

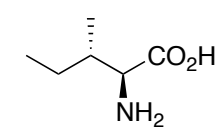

$(20 \mathrm{~mol} \%)$

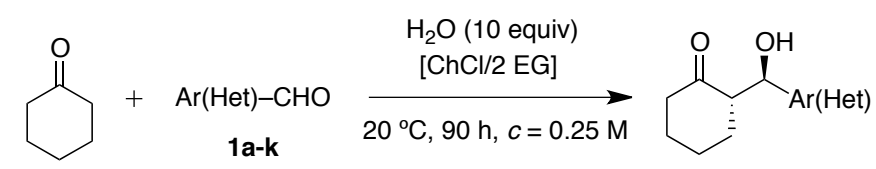

2a-k, 3

\begin{tabular}{|c|c|c|c|c|c|}
\hline entry & Ar- & 2 & yield $(\%)^{b}$ & $\mathrm{dr}(\text { anti/syn })^{c}$ & $e e(\%)^{d}$ \\
\hline 1 & 1a, $4-\mathrm{NO}_{2}-\mathrm{C}_{6} \mathrm{H}_{4}$ & $2 \mathbf{a}$ & $82(99)$ & $90: 10$ & 98 \\
\hline $2^{e}$ & $\mathbf{1 a}, 4-\mathrm{NO}_{2}-\mathrm{C}_{6} \mathrm{H}_{4}$ & $\mathbf{2 a}$ & (34) & $84: 16$ & 90 \\
\hline $3^{f}$ & $\mathbf{1 a}, 4-\mathrm{NO}_{2}-\mathrm{C}_{6} \mathrm{H}_{4}$ & $\mathbf{2 a}$ & 0 & - & - \\
\hline 4 & $\mathbf{1 b}, 3-\mathrm{NO}_{2}-\mathrm{C}_{6} \mathrm{H}_{4}$ & $\mathbf{2 b}$ & 75 & $93: 7$ & 97 \\
\hline $5^{g}$ & 1c, $2-\mathrm{NO}_{2}-\mathrm{C}_{6} \mathrm{H}_{4}$ & $2 \mathrm{c}$ & $80(99)$ & $90: 10$ & 99 \\
\hline 6 & 1d, $2,4-\mathrm{NO}_{2}-\mathrm{C}_{6} \mathrm{H}_{3}$ & $2 d$ & 68 & $93: 7$ & 96 \\
\hline 7 & 1e, $4-\mathrm{CN}-\mathrm{C}_{6} \mathrm{H}_{4}$ & $2 \mathrm{e}$ & 81 & $93: 7$ & 98 \\
\hline 8 & 1f, $2-\mathrm{CN}-\mathrm{C}_{6} \mathrm{H}_{4}$ & $2 f$ & 70 & $95: 5$ & 98 \\
\hline 9 & & $2 \mathrm{~g}$ & 82 & $88: 12$ & 97 \\
\hline 10 & $\mathbf{1 h}, 4-\mathrm{Cl}-\mathrm{C}_{6} \mathrm{H}_{4}$ & $2 h$ & 71 & $87: 13$ & 97 \\
\hline 11 & 1i, 4-Br- $\mathrm{C}_{6} \mathrm{H}_{4}$ & $2 \mathbf{i}$ & 66 & $93: 7$ & 98 \\
\hline 12 & $\mathbf{1 j}, 4-\mathrm{CO}_{2}-\mathrm{C}_{6} \mathrm{H}_{4}$ & $2 \mathbf{j}$ & 71 & $93: 7$ & 98 \\
\hline 13 & 1k, 2-pyridyl & $2 \mathbf{k}$ & 63 & $83: 17$ & 84 \\
\hline $14^{h}$ & $\mathbf{1 a}, 4-\mathrm{NO}_{2}-\mathrm{C}_{6} \mathrm{H}_{4}$ & & 72 & -- & 66 \\
\hline
\end{tabular}

${ }^{a}$ General reaction conditions: aldehyde $(0.2 \mathrm{mmol})$, cyclohexanone $(2.0 \mathrm{mmol})$, distilled water $(2.0 \mathrm{mmol})$ and L-isoleucine $(0.04 \mathrm{mmol})$ were added to the $[\mathrm{ChCl} / 2 \mathrm{EG}] \mathrm{DES}(0.8 \mathrm{~mL})$, at $20^{\circ} \mathrm{C}$. The reaction mixtures were stirred for $90 \mathrm{~h}$, and the reaction products were extracted with EtOAc. ${ }^{b}$ Isolated yield of analytically pure products 2 . In entries 1, 2 and 4 reaction conversion is given in brackets. ${ }^{c}$ Diastereisomeric ratio (dr) determined by ${ }^{1} \mathrm{H}$ NMR spectroscopy from crude reaction mixtures. ${ }^{d}$ Enantiomeric excess of major diastereoisomer (anti), as determined by chiral HPLC on analytically pure products. ${ }^{e}$ Control reaction carried out under analogous experimental conditions using pure EG as reaction medium. ${ }^{f}$ Blank experiment with no participation of isoleucine. ${ }^{g}$ Water was not added. ${ }^{h}$ Isobutyraldehyde (2-methylpropanal) was used instead of cyclohexanone, affording product 3a.

Challenging our system further, we next decided to carry out recycling experiments in which the aldehyde used as substrate would be changed after each catalytic run. Thus, after setting up a reaction between cyclohexanone and 4-nitrobenzaldehyde 1a (Table 3, cycle 1), the DES and L-isoleucine were recovered as stated in the previous paragraph. The so recycled material was then treated with cyclohexanone $(2 \mathrm{mmol})$ and 2-nitrobenzaldehyde $1 \mathrm{c}(0.2 \mathrm{mmol})$, being the mixture vigorously stirred for $90 \mathrm{~h}$, to render adduct 2c in quantitative conversion and excellent diastereoand enantioselectivity (Table 3 , cycle 2 ), with no traces of any product $\mathbf{2 a}$ coming from the previous run. It is worth noting that product $2 \mathrm{c}$ obtained by this way shows the same selectivity values as those reported in Table 1 , entry 5 , when it is prepared using fresh organocatalyst and DES. Finally, after recycling DES plus catalyst from the second cycle, a third reaction could be run within the same medium employing 4-nitrobenzaldehyde 1a (Table 3, cycle 3 ). Gratifyingly, the desired $\beta$-hydroxy ketone $2 \mathbf{a}$ was produced with comparable selectivities as those of Table 2 , entry 3 , and Table 1 , entry 1 , which refer to the same product. The aldol adduct $2 \mathrm{c}$ was not detected as a contaminant.

Table 3. Recyclability of DES and L-isoleucine in the asymmetric cross-aldol reaction between cyclohexanone and 4-nitrobenzaldehyde 1a, or 2-nitrobenzaldehyde 1c. ${ }^{a}$

\begin{tabular}{llllll} 
cycle & ArCHO & 2 & conv $(\%)^{b}$ & $\begin{array}{l}\mathrm{dr} \\
(\text { anti/syn })^{c}\end{array}$ & $\begin{array}{l}e e \\
(\%)^{d}\end{array}$ \\
\hline 1 & 1a & 2a & 99 & $90: 10$ & 98 \\
$2^{e}$ & 1c & 2c & 99 & $90: 10$ & 99 \\
3 & 1a & 2a & 99 & $88: 12$ & 98
\end{tabular}

${ }^{a}$ Reaction conditions: aldehyde $(0.2 \mathrm{mmol})$, cyclohexanone $(2.0 \mathrm{mmol})$, distilled water $(2.0 \mathrm{mmol})$ and $\mathrm{L}$-isoleucine $(0.04$ mmol) were added to $[\mathrm{ChCl} / 2 \mathrm{EG}](0.8 \mathrm{~mL})$, at $20{ }^{\circ} \mathrm{C}$. The reaction mixture was stirred for $90 \mathrm{~h}$. Recycling was performed as stated in the main text. ${ }^{b}$ Conversion of aldehydes $\mathbf{1 a}$ or $\mathbf{1 c}$ to anti/syn-2a or anti/syn-2c, as determined by ${ }^{1} \mathrm{H}$ NMR spectroscopy of crude reaction mixtures. Resonances of aldol adducts were integrated and quantified against $\mathrm{CHBr}_{3}(9 \mu \mathrm{L}, 0.103$ mmol) used as analytical internal standard. ${ }^{c}$ Diastereisomeric 
ratio (dr) determined by ${ }^{1} \mathrm{H}$ NMR spectroscopy from crude reaction mixtures. ${ }^{d}$ Enantiomeric excess of major diastereoisomer (anti), as determined by chiral HPLC on crude reaction mixtures. ${ }^{e}$ No water was used.

To the light of the experiments presented in Table 3, the reaction system DES-isoleucine keeps no memory when it is recycled and reused in further transformations. Therefore, the combination of L-isoleucine $+[\mathrm{ChCl} / 2 \mathrm{EG}]$ can be foreseen as a reaction kit for the asymmetric cross-aldol reaction between cyclohexanone and aromatic aldehydes, being reusable a minimum of three times with different substrates. Furthermore, the simplicity of the experimental setup, and the low cost of naturally-occurring L-isoleucine and DES, makes this methodology amenable of being adopted by any non-specialized group interested in getting access to compounds of the like of $\mathbf{2 a}-\mathbf{k}$.

To conclude, we have developed and implemented a novel L-isoleucine-based organocatalytic system for the cross-aldol reaction between cyclohexanone and aromatic aldehydes that operates in a $\mathrm{ChCl} / \mathrm{EG}$ DES. This protocol is green and straightforward. The reaction products can be isolated in high yield, and with good levels of diastereo- and enantioselectivity, comparable to those obtained by classical organocatalytic methodologies employing organic solvents. The amino acid catalyst and the reaction medium (DES) can be recycled a minimum of five times without altering the reaction outcome. This recycling process retains no memory about the substrate used, thus permitting the consecutive preparation of different aldol adducts with a single Lisoleucine-DES reaction kit. The development of other environmentally-friendly recyclable systems able to catalyze multiple organic transformations is currently underway in our laboratory, and will be reported in due course.

\section{ASSOCIATED CONTENT}

\section{Supporting Information}

General procedures, spectroscopic data, copies of ${ }^{1} \mathrm{H}$ and ${ }^{13} \mathrm{C}$ NMR spectra, and chromatographic data for compounds $\mathbf{2 a}-\mathbf{k}$, 3a.

\section{AUTHOR INFORMATION}

\section{Corresponding Author}

* Dr Vicente del Amo: Departamento de Química Orgánica e Inorgánica, Universidad de Oviedo, C/ Julián Clavería 8, 33006, Oviedo, Spain. E-mail: vdelamo@uniovi.es. Phone: + 3498510 3450. Fax: +34 985103446.

\section{ACKNOWLEDGMENT}

We thank MINECO (CTQ2014-55015-P) for financial support. C.C. expresses her gratitude to MINECO for the award of a "Ramon y Cajal" contract (RYC-2014-16021). The authors extend thanks to Dr Joaquín García-Álvarez (Universidad de Oviedo) for his useful advices along the preparation of the manuscript.

\section{REFERENCES}

${ }^{1}$ List, B.; Lerner, A. R.; Barbas, C. F., III. J. Am. Chem. Soc. 2000, 122, 2395-2397.
${ }^{2}$ Ahrendt, K. A.; Borths, C. J.; MacMillan, D. W. C. J. Am. Chem. Soc. 2000, 122, 4243-4244.

${ }_{3}^{3}$ (a) Anastas, P. T.; Warner, J. C. In Green Chemistry: Theory and Practice; Oxford University Press, New York, 1998. (b) Tang, S. L. Y.; Smith, R. L.; Poliakoff, M. Green Chem. 2005, 7, 761-762.

${ }^{4}$ Abbot, A. P.; Capper, G.; Davies, D. L.; Munro, H. L.; Rasheed, R. K.; Tambyrajah, V. Chem. Commun. 2001, 2010-2011.

${ }^{5}$ Hammond, O. S.; Bowron, D. T.; Edler, K. J. Green Chem. 2016, $18,2736-2744$

6 Abbot, A. P.; Capper, G.; Davies, D. L.; Rasheed, R. K.; Tambyrajah, V. Chem. Commun. 2003, 70-71.

${ }^{7}$ For some recent reviews on the use of DESs, with particular emphasis on their uses in organic transformations, see: (a) Guajardo, N.; Mueller, C. R.; Schrebler, R.; Carlesi, C.; Domínguez de María, P. Chem CatChem 2016, 8, 1020-1027. (b) Alonso, D. A.; Baeza, A.; Chinchilla, R.; Guillena, G.; Pastor, I. M.; Ramón, D. J. Eur. J. Org. Chem. 2016, 612-621. (c) García-Álvarez, J. Eur. J. Inorg. Chem. 2015, 31, 5147-5157. (d) Liu, P.; Hao, J.- W.; Mo, L.- P.; Zhang, Z.- H. RSC Adv. 2015, 5, 48675-48704. (e) Tang, B.; Row, K. H. Monatsh Chem. 2013, 144, 1427-1454. (f) Ruß, C.; König, B. Green Chem. 2012, 14, 2969-2982. (g) Zhang, Q.; De Oliveira Vigier, K.; Royer, S.; Jérôme, F. Chem. Soc. Rev. 2012, 41, 71087146.

${ }^{8}$ (a) Marset, X.; Pérez, J. M.; Ramón, D. J. Green Chem. 2016, 18, 826-833. (b) Vidal, C.; Merz, L.; García-Álvarez, J. Green Chem. 2015, 17, 3870-3878. (c) Rodríguez-Álvarez, M. J.; Vidal, C.; Díez, J.; García-Álvarez, J. Chem. Commun. 2014, 50, 12927-12929. (d) Vidal, C.; Suarez, F. J.; García-Álvarez, J. Cat. Commun. 2014, 44, 76-79.

${ }^{9}$ For a recent review, see: Potdar, M. K.; Kelso, G. F.; Schwarz, L.; Zhang, C.; Hearn, M. T. W. Molecules 2015, 20, 16788-16816.

${ }^{10}$ (a) Massolo, E.; Palmieri, S.; Benaglia, M.; Capriati, V.; Perna, F. M. Green Chem. 2016, 18, 792-797. (b) Martínez, R.; Berbegal, L.; Guillena, G.; Ramón, D. J. Green Chem. 2016, 18, 1724-1730. (c) Müller, C. R.; Meiners, I.; Domínguez de María, P. RSC Adv. 2014, 4, 46097-46101.

${ }^{11}$ (a) Martínez-Castañeda, A.; Poladura, B.; Rodríguez-Solla, H.; Concellón, C.; del Amo, V. Org. Lett. 2011, 13, 3032-3035. (b) Martínez-Castañeda, A.; Poladura, B.; Rodríguez-Solla, H.; Concellón, C.; del Amo, V. Chem. Eur. J. 2012, 18, 5188-5190. (c) Martínez-Castañeda, A.; Rodríguez-Solla, H.; Concellón, C.; del Amo, V. J. Org. Chem. 2012, 77, 10375-10381. (d) Martínez-Castañeda, A.; Kędziora, K.; Lavandera, I.; Rodríguez-Solla, H.; Concellón, C.; del Amo, V. Chem. Commun. 2014, 50, 2598-2560.

${ }^{12}$ For some recent monographs and reviews, see: (a) Modern Aldol Reactions; Mahrwald, R., Ed.; Wiley-VCH: Weinheim, 2004; Vols. 1 and 2. (b) Schetter, B.; Mahrwald, R. Angew. Chem., Int. Ed. 2006, 45, 7506-7525. (c) Geary, L. M.; Hultin, P. G. Tetrahedron: Asymmetry 2009, 20, 131-173.

13 (a) Mase, N.; Hayashi, Y. Compr. Org. Synth. 2014, 273-339. (b) Guilena, G.; Nájera, C.; Ramón, D. J. Tetrahedron: Asymmetry 2007, 18, 2249-2293.

${ }^{14}$ (a) Córdova, A.; Zou, W.; Ibrahem, I.; Reyes, E.; Engqvist, M.; Liao, W. W. Chem. Commun. 2005, 3586-3588. (b) Córdova, A.; Zou, W.; Dziedzic, P.; Ibrahem, I.; Reyes, E.; Xu, Y. Chem. Eur. J. 2006, 12, 5383-5397. (c) Hayashi, Y.; Itoh, T.; Nagae, N.; Ohkubo, M.; Ishikawa, H. Synlett 2008, 1565-1570. (d) Ma, G.; Bartoszewicz, A.; Ibrahem, I.; Córdova, A. Adv. Synth. Catal. 2011, 353, 3114-3122.

${ }^{15}$ The addition of some water was found to increase significantly the enantiomeric excess of the aldol products, in agreement with literature: Jimeno, C. Org. Biomol. Chem. 2016, 14, 6147-6164.

${ }^{16}$ Reaction time was set to $90 \mathrm{~h}$ to guarantee full conversion of aldehyde 1a into product $\mathbf{2 a}$. Conversion rises up under long reaction times at the expense of diastereoselectivity of aldol products 2 (Supporting Information, Table S8).

${ }^{17}$ Rohr, K.; Mahrwald, R. Org. Lett. 2012, 14, 2180-2183. 


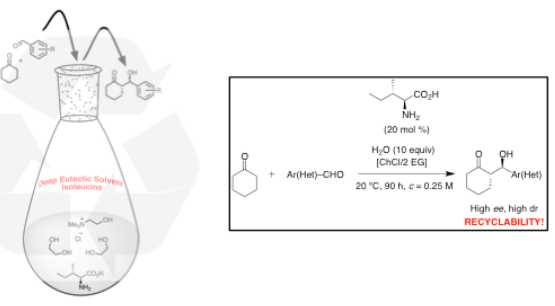

